\title{
SPACE-AERIAL-GROUND INTEGRATED CITY DYNAMIC MONITORING BASED ON REMOTE SENSING
}

\author{
Li CHEN ${ }^{1}$, Junyong HU' ${ }^{1}$, Guoliang LV ${ }^{1}$, Yunhe LIU ${ }^{1}$ \\ ${ }^{1}$ Shaanxi TIRAIN Science \& Technology Co. Ltd., Xi’an, Shaanxi, China \\ chen@trgis.com jyhu@trgis.com lvguoliang@trgis.com 412968856@qq.com
}

Commission III, WG III/1

KEY WORDS: Remote Sensing, change monitoring, artificial intelligence, three-dimensional real-scene

\begin{abstract}
:
With the further acceleration of the new urbanization process, China's urbanization construction has entered a new stage of more standardized and scientific development, which puts forward higher requirements for national land and space monitoring. In view of the rapid changes in looks of our cities, the periodic monitoring methods such as personnel patrolling inspection, public reporting or satellite remote sensing are difficult to meet the needs of full-range, dynamic and precise monitoring. Therefore, it is urgent to establish a new spatial monitoring system to make up for the deficiencies of current urban spatial monitoring. In the article, the authors provide an implementation idea of a space-aerial-ground integrated remote sensing monitoring method system, which integrates various remote sensing techniques and information platform to meet the needs of large-scale urban change cycle monitoring and continuous monitoring of key construction areas Space monitoring provides modern technical support. Some of the techniques in the method system described here have been applied in some regions, which provides a basic platform for regional city monitoring, and have further research and application prospects.
\end{abstract}

\section{INTRODUCTION}

With the implementation of major national strategies such as new urbanization construction and a new round of territorial and spatial planning, China's urbanization process is accelerating and its cities are changing with each passing day. In the past, due to the lack of effective supervision methods, along with the rapid development of urban construction, such as the growth of urban borders and the transformation of shantytowns, various illegal activities have emerged one after another including occupying land or even crossing the redline. In the past, the passive management mode relied on manual inspections and the people report. It lacked technical means to perceive continuous, large-scale, and refined changes in urban space, and was unable to curb various types of violations in the first time. This not only increases the governance cost on demolition and renovation, and may cause a huge social and economic losses, also easy to stimulate social contradictions in a wide range, which seriously restricts the government's ability to manage urban space and improve the service level for the public.

In recent years, many scholars at home and abroad have studied the satellite imagery for space monitoring from different perspectives. Academician Dr. Li Deren applied remote sensing in humanitarian assessment, spatial and temporal pattern analysis of urban development, and many other areas(LI Deren,2017; LI Deren 2018); Paola Benedetti et al. proposed a deep learning architecture based on multi-scale and multitemporal satellite data fusion to study land cover(Benedetti P,2019); Li Kangning et al. proposed a method for extracting urban distribution and urban space development dynamics using remote sensing(Kangning L,2018); In addition, more scholars have applied remote sensing technology to different fields including agriculture, forestry, and ecology, monitoring growth, area and other information(Zhen CHEN,2019;Luigi R,2018;Liu $\mathrm{SH}, 2017)$. These studies show that the remote sensing has wide application value in the special monitoring of large areas for urban ecology, urban structures, agriculture and forestry. Here the authors attempt to explore a more automated and intelligent method system in terms of urban spatial changes. With this goal in mind, we study the automatic extraction of urban spatial changes from two aspects: intelligent comparison of multiperiod remote sensing imagery changes and automatic analysis of building changes in local areas. With regards to intelligent comparison of multi-period remote sensing imagery, we propose a method of extracting building information from imageries of different time phases, and further using the extracted information to support the coordinate correction of multi-phased imagery, and then comparing a the changing areas. Among them, in the aspect of automatic information extraction of remote sensing imageries, various methods are mainly based on imagery texture features to calculate, such as Zhu Jinshan's proposed high-resolution remote sensing image building extraction method based on MNF transformation(Zhu JS,2019). In addition, many scholars have also applied convolutional neural networks in the extraction of remote sensing information. Through pixel segmentation and sample library establishment, the effect of building recognition has been improved (Ren XL,2019;Cai SK,2017). In the building extraction in the paper here, an artificial intelligence algorithm based on convolutional neural network is directly applied. By finding the same key features in different images, it provides a basis for calibrating different images into a unified coordinate system. For the automatic analysis of building changes in local areas, the image edge detection technique is adopted to compare the existing model projection image with the live image collected in real time to find the difference. Image edge detection is a popular research field in image feature extraction(Wang MJ,2011). These studies provide authors more theoretical references for the dynamic monitoring of urban spatial remote sensing. In this paper, from the perspective of comprehensively sensing the dynamic changes of urban space and building an information platform, based on the "space-aerial-ground" full-sequence remote sensing technology, the use of deep learning and real-

* Guoliang LV (lvguoliang@trgis.com) is the Corresponding author. 
time analysis of three-dimensional real-scene and other technologies to realize the overall solution of intelligent and automated monitoring of urban space changes, providing the necessary technology and platform support for the global, fulltime, full-process informationization and intelligent monitoring of urban space utilization.

\section{RESEARCH CONTENT}

Three-dimensional sensing methods including space remote sensing, aerial remote sensing, and ground remote sensing provide multi-scale, reliable, objective, and efficient data sources for urban space monitoring from the global to the local level, which are the prerequisites for urban space monitoring. In the past, there has been a long processing cycle for the analysis and utilization of these data and the monitoring methods are mostly limited to visual interpretation, which greatly reduces the timeliness of data use. Due to the time-consuming and labour-intensive processing process, large costs are also consumed. This paper focuses on the construction of a realscene three-dimensional platform for urban space monitoring using remote sensing and its key technologies, monitoring process, long-term mechanism construction and other research results, providing information solutions for the whole process of urban space monitoring from data management, intelligent processing to monitoring analysis.

\subsection{Intelligent Dynamic Three-dimensional Real-Scene Monitoring Platform}

The main technical contents of the intelligent dynamic threedimensional real-scene monitoring platform for urban space include four major aspects: 1. Establish an automatic correction system for remote sensing image data such as satellites and drones; 2. Establish an intelligent contrast analysis system for remote sensing image data; 3 . Establish a $3 \mathrm{D}$ visualization system of urban real scene based on oblique photography; 4. Establish a mobile monitoring system for illegal structures. The automatic correction system of remote sensing image data realizes the automatic correction of remote sensing image through artificial intelligence technology. The intelligent contrast analysis system of remote sensing image data extracts the change map spots by automatically comparing and analyzing multi-phase images in the same area, and realizes the monitoring of urban spatial changes in the entire region. The three-dimensional urban real-time visualization system integrates a variety of spatial data from satellites and unmanned aerial vehicles tilt photography to form urban digital twin models, providing a visual platform support for urban space monitoring, research and judgment. The illegal construction mobile monitoring system is based on urban digital twin models On the other hand, by using video images around the road, it can quickly analyze the changes in the surrounding buildings and provides technical support for real-time tracking and monitoring of key areas.

The three-dimensional real-scene monitoring platform is the core platform of the entire monitoring process. The threedimensional Real-scene monitoring platform realizes information-based support for the entire process of urban space monitoring, including management of various data sources, automatic correction of the latest remote sensing data, intelligent detection of multi-period remote sensing data changes, determination of suspected speckles, and visual command and decision-making. It provides a modern monitoring method for urban space monitoring, using "spaceaerial-ground" remote sensing as the sensing means, artificial intelligence interpretation as the core of technology, and threedimensional visualization as the display platform. The real-time monitoring platform now supports the loading of highresolution image data and various model data, and supports the $3 \mathrm{D}$ visualization display of monitoring results. In the framework design, the platform adopts component-based design to support the integration of algorithm libraries such as multi-phase image comparison and scene comparison analysis, so as to provide a basic framework for continuously improving the platform's monitoring capability.

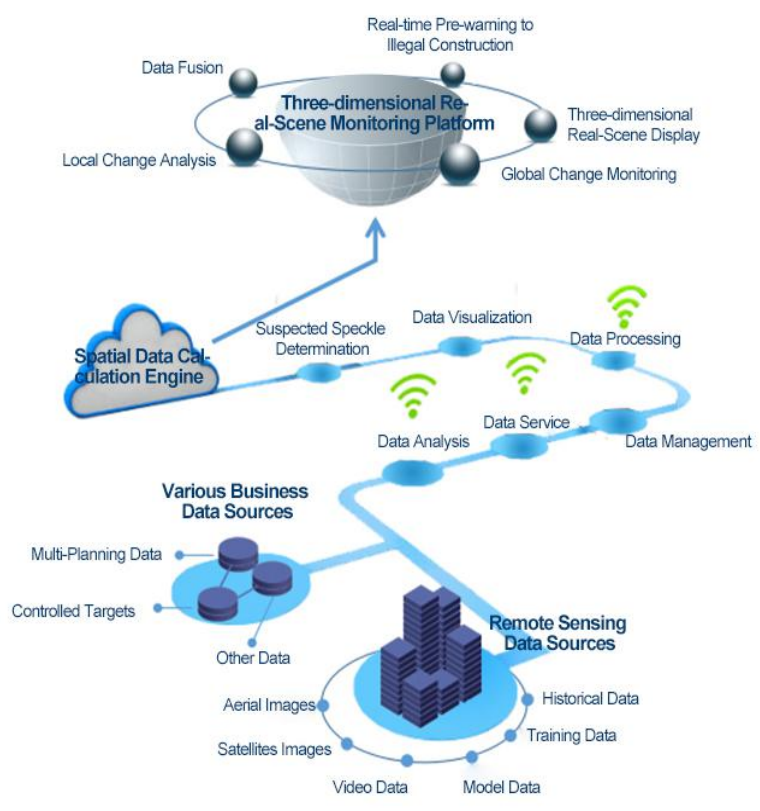

Fig.1 System Structure

\subsection{Monitoring Flowchart}

The urban space monitoring method in this paper combines satellite monitoring, unmanned aerial vehicles monitoring, vehicle-mounted video mobile monitoring, and personnel inspection, among which, satellite monitoring can adopt multisource satellite data of China's Gaofen (high-resolution) series and Gaojing (high-view) series to conduct remote sensing monitoring for urban-wide spatial coverage twice a year. For some key areas, drones can be used for quarterly remote sensing monitoring. For core construction areas, mobile vehiclemounted with video, position and posture acquisition devices can be used for weekly routine monitoring. The specific monitoring process consists of the following parts:

\section{(a) Satellite Monitoring}

Through the system to input the latest satellite remote sensing data, these remote sensing data are first automatically matched and corrected by the system to form fully matched comparison data with historical images, and then the latest data are automatically compared with historical images to generate change patches on map.

\section{(b) Unmanned Aerial Vehicles Monitoring}

Unmanned aerial vehicle (UAV) monitoring includes periodic monitoring and mobile monitoring. Mobile monitoring is mainly to respond to the suspected changes found from the 
satellite images which need to be confirmed further. Through the periodic monitoring and mobile monitoring, a more timely monitoring effect is achieved. The processing of UAV monitoring is similar to that of satellite monitoring.

\section{(c) Vehicle-mounted Video Mobile Monitoring}

Vehicle-mounted video mobile monitoring includes periodic monitoring and mobile monitoring. Through the vehiclemounted video mobile monitoring, the timeliness of monitoring is further improved. In addition, due to the method of the stereoscopic contrast analysis with the real-scene models, the spatial monitoring on both sides of the road can be more refined.

\section{(d) Personnel Inspection}

In combination with that three automatic monitoring modes of the satellites, the unmanned aerial vehicle and patrol vehicle, the urban spatial changes have been systematically screened and focused, and the change areas screened out are supplemented by manual verification. Such information can be shared and pushed to various professional departments of urban management through the information platform, providing a basis for the rapid processing by relevant departments.

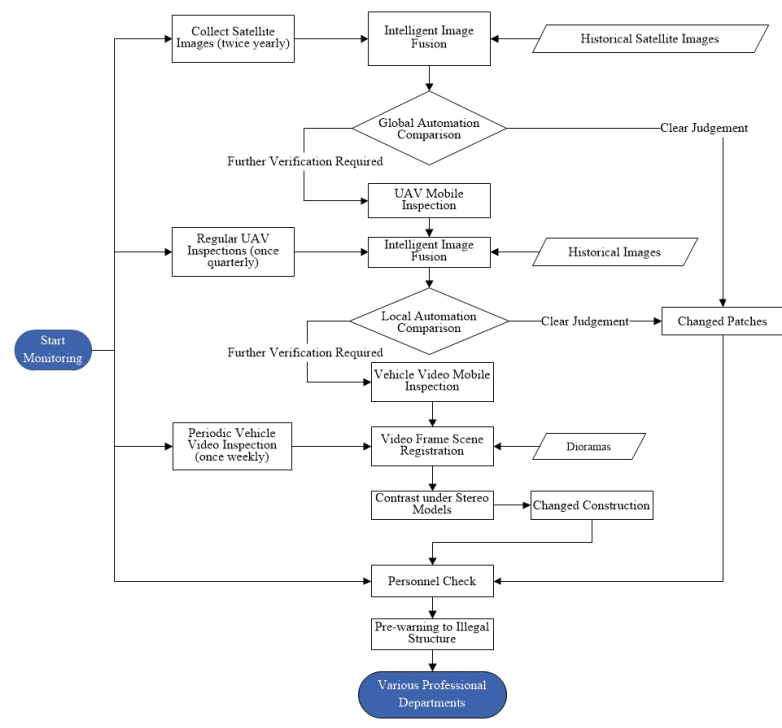

Fig.2 Monitoring Workflow

\subsection{KEY TECHNOLOGIES}

The key technologies of the urban spatial intelligent dynamic three-dimensional real-scene monitoring platform mainly include three aspects: the three-dimensional real-scene visualization technology, image-based contrast analysis technology, and video-based contrast analysis technology. The three-dimensional real-scene visualization technology provides spatial graphics support for comparison analysis and result display of images and videos. The image-based contrast analysis technology realizes the rapid comparison and analysis of aerospace remote sensing images from different phases and extracts inconsistent patches on images to monitor urban spatial changes at a more macro level; The video-based comparison analysis has realized the high-precision change detection of the local areas on both sides of the road by the video collected by the mobile patrol vehicle, and urban spatial changes can be monitored more accurately from a micro level. Through the organic integration of these technologies, a more comprehensive intelligent technology system for urban space monitoring is formed.

\subsubsection{Three-dimensional Real-scene Visualization Technology}

The three-dimensional real-scene visualization technology adopts the three-dimensional engine independently developed by TIRAIN Technologies to achieve the processing, management, service release, and 3D rendering of digital elevation model (DEM), digital orthophoto map (DOM), model data, and point cloud data, which supports online loading and visualization of various spatial data and supports integration and mounting of various non-spatial data such as panorama, video, text, etc.. It also provides various spatial analysis algorithms such as spatial distance, area, slope, aspect, earthwork, geological section, and visibility, as well as various spatial simulation algorithms for sunlight, immersion, excavation, and equality. It provides support for the integration of various types of data, as well as comprehensive operations and visualization output of results.

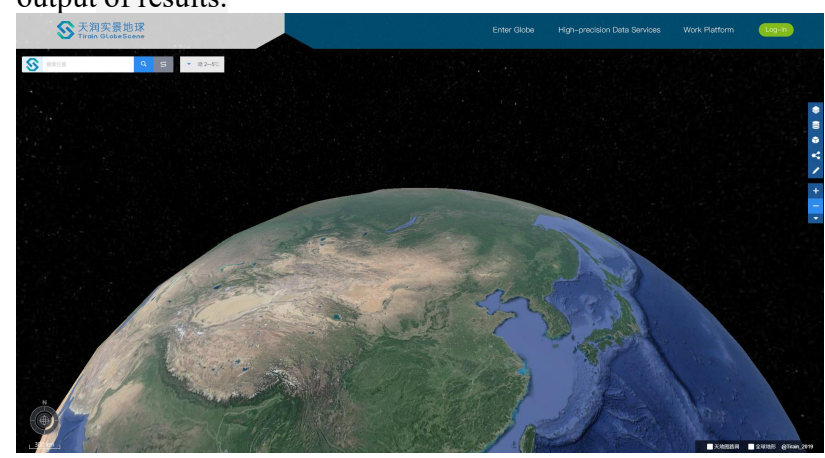

Fig.3 TIRAIN 3D Real-Scene Monitoring Platform

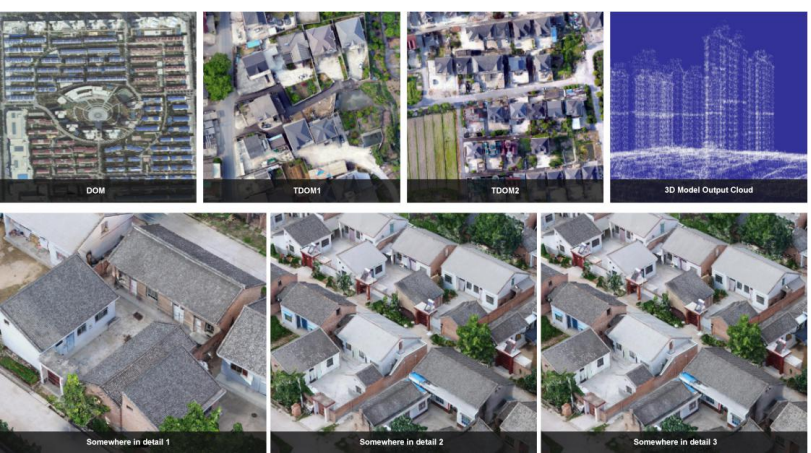

Fig.4 TIRAIN 3D Real-Scene Monitoring Platform

\subsubsection{Image-based Fast Extraction for Inconsistent Speckles}

As an easily available, objective and realistic data source, remote sensing images provide a basis for urban space monitoring. However, the direct use of remote sensing images for manual comparison obviously cannot meet the needs of large-scale and efficient monitoring. In order to improve the monitoring efficiency using remote sensing images, during the construction of the platform, we have solved the two major problems of automatic image correction and automatic image comparison, and formed an image-based fast extraction technology of inconsistent image speckles. These two major algorithms of automatic extraction technology are introduced 
below. It consists of three parts: target recognition based on high-resolution remote sensing image, image correction based on target, and patch extraction based on inconsistency.

(a) Target recognition based on high-resolution remote sensing images. The image segmentation method is used to segment the acquired remote sensing image, generate the corresponding target patch and use it as the basic analysis unit, and train the deep network model according to the sample library of different feature categories, as shown in the figure, improve the classification accuracy and robustness of the network, and complete the terrain recognition task of high-resolution remote sensing image.

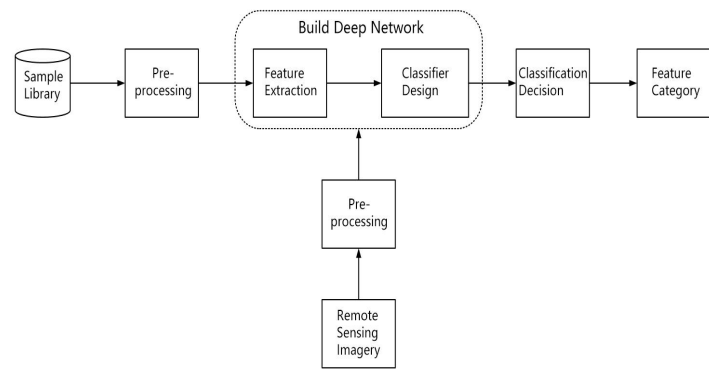

Fig. 5 Classification framework of high-resolution remote sensing images

(b) Fast correction algorithm based on sequence image. (1) In the selected area, the above-mentioned target recognition algorithm is applied to the historical image and the image to be corrected simultaneously to extract feature elements; (2) the better identical features (the same object in different images) are extracted from the historical image and the image to be corrected for calibration; (3) the corresponding points of the images are automatically established and more are added to make it dense enough according to the identical elements to calculate the correction matrix; (4) Matrix operation is carried out on the image to be corrected to form the corrected results, which provides the data basis for the extraction of inconsistent image patches.

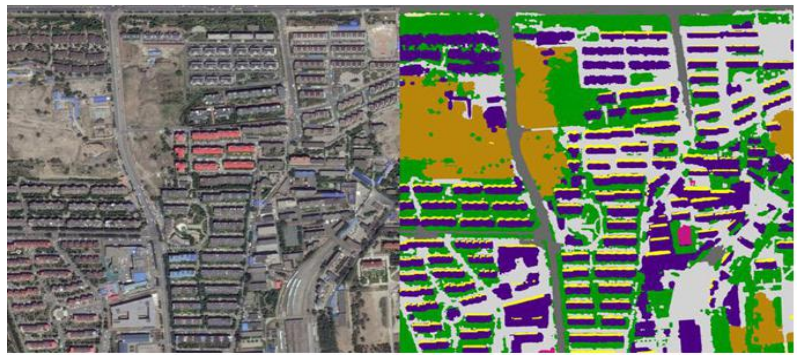

Fig.6 Features Extraction Based on Machine Learning

(c) Automatic extraction algorithm for inconsistent images patches. (1) Set the minimum unit of the pixel block, such as a pixel unit of 5 pixels by 5 pixels; (2) Due to various differences in the shooting and processing of historical images and comparison images, the pixel values of the corresponding pixels in the two images may be inconsistent. Before performing image pixel comparison, it is necessary to establish a test function of the corresponding pixels between the two images; (3) Construct a corresponding pixel block function by using the corresponding pixel test function and a given threshold value (the noise points of individual pixels in the pixel block are excluded by the threshold value); (4) Through the corresponding pixel block function, the two images are compared to form an inconsistent image patch extraction result.
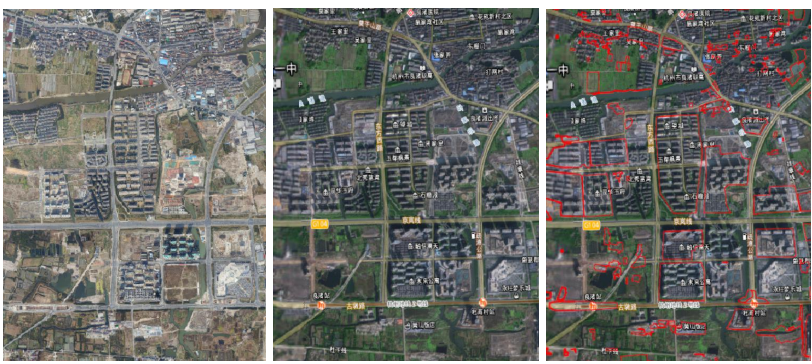

Fig. 7 Images in the pilot area in 2017 \& 2018 and inconsistencies extraction

\subsubsection{Video-based Architectural Inconsistent Information Automatic Extraction Technology}

Both sides of the road are usually the focus of urban space monitoring. The video-based automatic extraction of architectural inconsistent information is based on the comparison of real-scene models obtained by oblique photography, and an algorithm is used to automatically compare the video information with the model, then the inconsistent information of the comparison is automatically marked on the models and videos, providing us with a fast and automated means of monitoring building changes. The main implementation steps of this technology are as follows:

(a) The mobile patrol vehicles equipped with position, attitude and image sensors are used to collect realtime video images with position and attitude;

(b) Register each frame of the video in the threedimensional real-scene platform in real time according to the position and attitude (or posture);

(c) The three-dimensional real-scene platform forms a projection image corresponding to each frame of the video by controlling various parameters of the scene camera according to the information registered in the video frame;

(d) Automatically label the architectural outline of the projected image based on the three-dimensional building model, and generate the building outline through the three-dimensional models;

(e) Perform the edge detection on single-frame video images, compare the scene footprint of the projected image with the corresponding outline of the singleframe video images, and quickly find the differences, so as to monitor whether there is the unauthorized construction to the existing ones or not;

\section{TYPICAL APPLICATION FIELDS OF REMOTE SENSING URBAN SPACE MONITORING}

The "space-aerial-ground" integrated urban space dynamic monitoring platform based on remote sensing has great application value to the current space utilization, urban 
management, ecological governance and other aspects: In terms of space utilization, remote sensing monitoring can provide the current monitoring data reference for the urban space planning. On the other hand, remote sensing monitoring can also provide support for the urban space "One-sheet of Map" monitoring and early warning of. In terms of urban management, with the help of remote sensing monitoring, various illegal events such as illegal construction and illegal land occupation can be found, and the intelligent perception ability of the overall operating status of urban space can be improved; In terms of the ecological environment protection, with the help of remote sensing monitoring, the ecological monitoring and effectiveness evaluation of the city-wise can be realized. The integrated " space-aerial-ground " urban space dynamic monitoring platform based on remote sensing will empower the comprehensive perception of regional space and escort the sustainable development of the region.

\section{APPLICATION OF REMOTE SENSING URBAN SPACE MONITORING}

The urban space remote sensing intelligent dynamic monitoring platform described in this paper has been applied to a certain range in Zhejiang, Hainan, Shaanxi and other places. Through the application of the platform, the level of urban space monitoring in these areas has been greatly improved, enabling the relevant departments to timely control and stop various illegal acts such as illegal construction and illegal land occupation, thus saving the urban governance costs and preventing the unnecessary social funds waste.

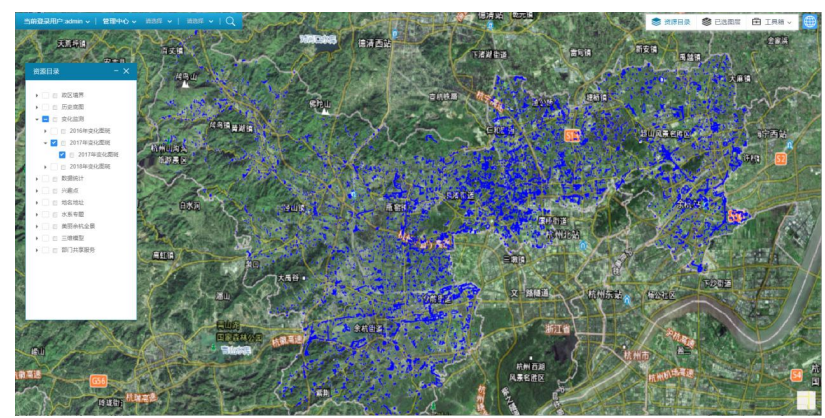

Fig.8 Unban Space Change Monitoring Result for Yuhang District in 2017

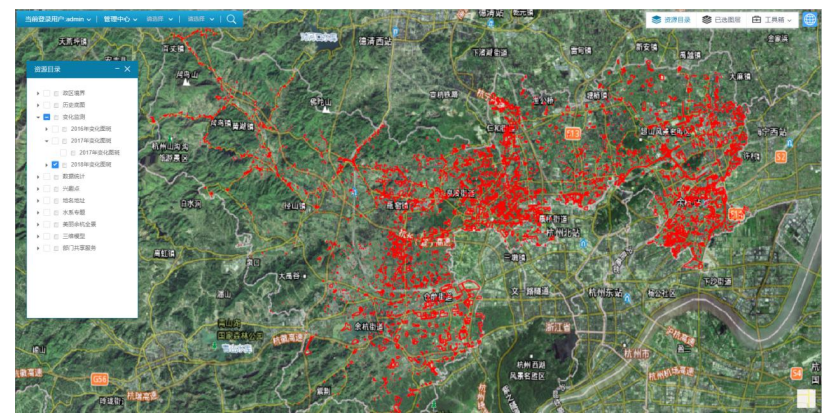

Fig.9 Unban Space Change Monitoring Result for Yuhang District in 2018

\section{CONCLUSIONS}

In this paper, the authors provide the research ideas for informatization platform and key technology for large-area urban space dynamic monitoring and accurate monitoring of building changes in key areas based on remote sensing technology. And the key technologies such as the remote sensing-based urban spatial dynamic monitoring visualization platform, remote sensing image-based dynamic extraction of urban changes and video-based building change detection have been studied. At present, the main function development of the urban space dynamic monitoring visualization platform has been completed, which supports the three-dimensional display of various spatial monitoring data and monitoring results. In terms of algorithm research, building extraction based on highresolution remote sensing images has been achieved, with an accuracy rate of $94 \%$. Follow-ups will be based on the results of recognition for further research, automatic calibration of test images, intelligent comparison and related algorithms of scene comparison. The research results obtained by the platform have been deployed and tested in some regions, providing platform support for regional space monitoring. Next, we will continue to enrich the platform's intelligent monitoring items and get through all the key technologies described in this article. We will continue to carry out more extensive verification in the test area to provide a more complete platform for comprehensive remote sensing services.

\section{REFERENCES}

Benedetti P , Ienco D , Gaetano R , et al. M3 Fusion: A Deep Learning Architecture for Multi-\{Scale/Modal/Temporal $\}$ satellite data fusion[J]. IEEE Journal of Selected Topics in Applied Earth Observations and Remote Sensing, 2019, 11(12):4939-4949.

Cai SK, Liu JQ, Shi WZ, et al. High resolution remote sensing image building extraction based on improved SLIC and regional adjacency map [J]. Computer system application, 2017 (8)

Kangning L , Yunhao C . A Genetic Algorithm-Based Urban Cluster Automatic Threshold Method by Combining VIIRS DNB, NDVI, and NDBI to Monitor Urbanization[J]. Remote Sensing, 2018, 10(2):277-

LI Deren, LI Xi. Use of night-time light remote sensing in humanitarian disaster evaluation[J]. Chinese Journal of Nature,2018, 40(3)

LI Deren, YU Hanruo, LI Xi. The Spatial-Temporal Pattern Analysis of City Development in Countries along the Belt and Road Initiative Based on Nighttime Light Data[J]. Geomatics and Information Science of Wuhan University,2017, 42(6)

Liu SH , Yang YP, Zhao ZH , et al. Application requirement analysis of high spectral and high spatial resolution satellite for environment remote sensing monitoring[C]// Society of Photooptical Instrumentation Engineers. Society of Photo-Optical Instrumentation Engineers (SPIE) Conference Series, 2017.

Luigi R , Elisa C , Mirco B , et al. Assessment of Water Management Changes in the Italian Rice Paddies from 2000 to 2016 Using Satellite Data: A Contribution to Agro-Ecological Studies[J]. Remote Sensing, 2018, 10(3):416-. 
Ren XL, Wang YP, Yang JY, et al. Remote sensing image building extraction based on improved u-net [J]. Progress in laser and optoelectronics, 2019, 56 (22)

Wang MJ, Yang TW, Han JD, et al. Overview of image edge detection technology [J]. Journal of Central South University: Natural Science Edition, 2011, 42 (S1): 811-816

Zhen CHEN,Yunshi ZHANG,Yuanyu ZHANG,et al A study of remote sensing monitoring methods for the high standard farmland[J],National Land \& Resources Remote Sensing 2019, $31(2)$

Zhu JS, Song ZZ, Ji XY. High resolution remote sensing image building extraction method based on MNF transform [J]. Mapping and spatial geographic information, 2019 (7): 8-10 